\title{
On Maxwellian equilibria of insulated semiconductors
}

\author{
LUIS CAFFARELLI \\ Department of Mathematics, University of Texas at Austin, RLM 8100, Austin, TX 78712, USA \\ JEAN DOLBEAULT \\ CEREMADE, Université de Paris IX-Dauphine, Place du Maréchal de Lattre de Tassigny, \\ 75775 Paris Cedex 16, France \\ Peter A. MARKOWICH \\ Institut für Mathematik, Universität Wien, Boltzmanngasse 9, 1090 Wien, Austria \\ AND \\ CHRISTIAN SCHMEISER \\ Institut für Angewandte und Numerische Mathematik, TU Wien, Wiedner Hauptstraße 8-10, \\ 1040 Wien, Austria
}

[Received 17 September 1999]

\begin{abstract}
A semi-linear elliptic integro-differential equation subject to homogeneous Neumann boundary conditions for the equilibrium potential in an insulated semiconductor device is considered. A variational formulation gives existence and uniqueness. The limit as the scaled Debye length tends to zero is analysed. Two different cases occur. If the number of free electrons and holes is sufficiently high, local charge neutrality prevails throughout the device. Otherwise, depletion regions occur, and the limiting potential is the solution of a free boundary problem.
\end{abstract}

Keywords: Semiconductors, equilibrium, free boundary problems, charge neutrality

\section{Introduction}

We consider a bounded domain $\Omega \subset \mathbb{R}^{d}$ representing a piece of semiconductor. Built into the semiconductor crystal are impurities creating a fixed distribution of background charges $C(x), x \in$ $\Omega$, called the doping profile. In addition, the crystal contains $N$ (negatively charged) free electrons and $P$ (positively charged) holes both of which can move freely within $\Omega$. We assume global charge neutrality:

$$
N-P-\int_{\Omega} C \mathrm{~d} x=0
$$

We consider equilibrium states where the local distributions of electrons $n(x)$ and holes $p(x)$ are, according to the Boltzmann-Maxwell statistics,

$$
n(x)=\frac{N \exp (q \varphi(x) /(k T))}{\int_{\Omega} \exp (q \varphi /(k T)) \mathrm{d} x}, \quad p(x)=\frac{P \exp (-q \varphi(x) /(k T))}{\int_{\Omega} \exp (-q \varphi /(k T)) \mathrm{d} x} \quad x \in \Omega .
$$

Here $\varphi$ denotes the electrostatic potential, and the parameters $q, k, T$ are, respectively, the elementary charge, the Boltzmann constant, and the temperature of the crystal lattice. This form 
of the distributions can be derived from the equilibrium condition for different transport models such as the drift-diffusion model (see, e.g. [10]), the Vlasov equation [4, 5], or the Vlasov-PoissonBoltzmann equation [3]. In the context of semiconductors, mostly the case of devices with contacts, resulting in the absence of the integral terms in the formulas for the densities, has been previously considered.

The electrostatic potential is determined from the Poisson equation

$$
\varepsilon \Delta \varphi=q(n-p-C), \quad \text { in } \Omega,
$$

where $\varepsilon$ denotes the permittivity of the semiconductor. As a model for an insulated device we consider homogeneous Neumann boundary condidtions:

$$
\frac{\partial \varphi}{\partial v}=0, \quad \text { on } \partial \Omega .
$$

Note that the global neutrality assumption (1.1) is necessary for the solvability of the Neumann problem (1.2), (1.3).

For a non-dimensionalization the reference length $L$ (say, the diameter of $\Omega$ ) and the reference particle density $\tilde{C}$ (a typical value of the doping profile) are chosen. Then the potential is scaled by the thermal voltage $k T / q$ and the electron and hole numbers $N$ and $P$ by $L^{3} \tilde{C}$. We obtain the dimensionless equations

$$
\begin{aligned}
& \lambda^{2} \Delta \varphi=n-p-C, \quad \text { with } \\
& n=\frac{N \exp (\varphi)}{\int_{\Omega} \exp (\varphi) \mathrm{d} x}, \quad p=\frac{P \exp (-\varphi)}{\int_{\Omega} \exp (-\varphi) \mathrm{d} x},
\end{aligned}
$$

where the dimensionless parameter

$$
\lambda=\frac{1}{L} \sqrt{\frac{\varepsilon k T}{q^{2} \tilde{C}}}
$$

can be interpreted as the scaled Debye length. For notational simplicity, dimensionless quantities are denoted by the same symbols as their dimensional counterparts. The dimensionless versions of the global neutrality assumption and of the boundary conditions keep their forms (1.1) and (1.3), respectively.

Unique solvability of the problem (1.3)-(1.5) for the potential (up to an additive constant) has been proven in [19] (for a class of equilibrium distributions including the BoltzmannMaxwell statistics). Similar results can be found in [18], where mixed Dirichlet-Neumann boundary conditions are considered, and in [4] and [5], where instead of a bounded domain a confining potential is used. For the sake of completeness the proof is outlined and the result is formulated in Section 2. The basic idea is to rewrite the problem in variational form.

Our main interest is the limit $\lambda \rightarrow 0$. The formal limit of the Poisson equation (1.4) is the local neutrality equation

$$
0=n-p-C,
$$

implying $n \geqslant C_{+}, p \geqslant C_{-}$(where $C_{ \pm}$denote the absolute values of the positive and negative parts, i.e., $C=C_{+}-C_{-}$). Integration shows that this is only possible for

$$
N \geqslant \int_{\Omega} C_{+} \mathrm{d} x, \quad P \geqslant \int_{\Omega} C_{-} \mathrm{d} x
$$


These two conditions are equivalent under the global neutrality assumption (1.1). In [19], the limit (1.6) has been justified under the assumption (1.7). A simplified proof for BoltzmannMaxwell statistics is given in Section 2. This can be seen as an extension of earlier results for devices with contacts $[9,10]$.

If (1.7) is violated, local neutrality cannot hold throughout $\Omega$. This case is analysed in Section 3 . The rescaled potential $\Phi=\lambda^{2} \varphi$ is introduced, and it is shown that its limit satisfies a free boundary problem. The free boundaries separate depletion regions (where $n=p=0$ holds) from charge neutral regions (where (1.6) holds). The limiting potential solves a double obstacle problem where the difference between the obstacles is determined by a side condition. As in the full problem, the limiting problem possesses a variational formulation. Depletion regions have already been part of the pioneering theoretical investigations of semiconductor devices by Shockley [17]. Mathematically, free boundary problems have been derived as singular limits of the classical drift-diffusion model $[1,2,6,14-16]$ and they occur in the solution of drift-diffusion models with nonlinear diffusion $[7,11]$. Also, we refer to [12] and [13], where the theory of viscosity solutions is used to pass to the limit $\lambda \rightarrow 0$.

\section{Existence, uniqueness, and the charge neutral limit}

For functions $\psi \in \mathcal{H}=\left\{\psi \in H^{1}(\Omega): \int_{\Omega} \psi \mathrm{d} x=0\right\}$ we introduce the functional

$$
J(\psi)=\int_{\Omega}\left(\frac{\lambda^{2}}{2}|\nabla \psi|^{2}-C \psi\right) \mathrm{d} x+N \ln \left(\int_{\Omega} \mathrm{e}^{\psi} \mathrm{d} x\right)+P \ln \left(\int_{\Omega} \mathrm{e}^{-\psi} \mathrm{d} x\right) .
$$

In the following it will be shown that $J$ is coercive, bounded from below, and strictly convex. Since $\mathrm{e}^{z}$ and $\mathrm{e}^{-z}$ are convex functions, Jensen's inequaltity gives

$$
\exp \left( \pm \frac{1}{|\Omega|} \int_{\Omega} \psi \mathrm{d} x\right) \leqslant \frac{1}{|\Omega|} \int_{\Omega} \mathrm{e}^{ \pm \psi} \mathrm{d} x
$$

implying

$$
\begin{aligned}
J(\psi) & \geqslant \frac{\lambda^{2}}{2} \int_{\Omega}|\nabla \psi|^{2} \mathrm{~d} x+\int_{\Omega} \frac{N-P-|\Omega| C}{|\Omega|} \psi \mathrm{d} x+(N+P) \ln |\Omega| \\
& \geqslant \frac{\lambda^{2}}{2}\|\nabla \psi\|_{L^{2}(\Omega)}^{2}-c_{2}\|\psi\|_{L^{2}(\Omega)}-c_{3} \\
& \geqslant \frac{\lambda^{2}}{2}\|\nabla \psi\|_{L^{2}(\Omega)}^{2}-c_{4}\|\nabla \psi\|_{L^{2}(\Omega)}-c_{3}
\end{aligned}
$$

where the constants $c_{j}$ may depend on $\lambda^{2}, N, P,|\Omega|$, and $\|C\|_{L^{2}(\Omega)}$. The last inequality is a consequence of the Poincaré inequality $\|\psi\|_{L^{2}(\Omega)} \leqslant c_{5}\|\nabla \psi\|_{L^{2}(\Omega)}$, holding for every $\psi \in \mathcal{H}$. By (2.1), $J$ is coercive and bounded from below.

Since the first term in $J$ is strictly convex on $\mathcal{H}$, it suffices to prove convexity of the second and third terms. For $0<t<1$, the Hölder inequality gives

$$
\int_{\Omega} \mathrm{e}^{ \pm t \psi_{1} \pm(1-t) \psi_{2}} \mathrm{~d} x \leqslant\left(\int_{\Omega} \mathrm{e}^{ \pm \psi_{1}} \mathrm{~d} x\right)^{t}\left(\int_{\Omega} \mathrm{e}^{ \pm \psi_{2}} \mathrm{~d} x\right)^{1-t} .
$$


Taking the logarithm proves convexity of the second and third terms in $J$. Weak lower semicontinuity of $J$ is immediate.

A consequence of our results is the existence of a unique minimizer $\varphi \in \mathcal{H}$ of the functional $J$. The variation of $J$ is easily computed. Using the global neutrality assumption (1.1), it is straightforward to show that a criterion for the minimization is the weak formulation of the problem (1.3)-(1.5) together with the condition $\int_{\Omega} \varphi \mathrm{d} x=0$. When removing this last condition, the solution can obviously be shifted by an arbitrary additive constant. Regularity of the solution is the consequence of elliptic regularity results. Consequently, we obtain Theorem 2.1

THEOREM 2.1 Let the domain $\Omega \subset \mathbb{R}^{d}, 1 \leqslant d \leqslant 3$, have a smooth boundary and let it satisfy $0<|\Omega|<\infty$. Let $C \in L^{\infty}(\Omega)$ and (1.1) hold. Then, problem (1.3)-(1.5) has a solution in $W^{2, p}(\Omega)$ for every $p<\infty$, which is unique up to an additive constant.

We are going to carry out the limit $\lambda \rightarrow 0$. We proceed in several steps. First, we use the freedom of choosing an arbitrary additive constant in the potential. From now on the potential is chosen such that

$$
\frac{N}{\int_{\Omega} \exp (\varphi) \mathrm{d} x}=\frac{P}{\int_{\Omega} \exp (-\varphi) \mathrm{d} x}=: n_{i},
$$

and, thus, $n=n_{i} \mathrm{e}^{\varphi}, p=n_{i} \mathrm{e}^{-\varphi}$. The parameter $n_{i}$ (in the equilibrium condition $n p=n_{i}^{2}$ ) is called the intrinsic density in the semiconductor literature. The uniform boundedness of the densities will now be shown.

Lemma 2.2 Let the assumptions of theorem 2.1 hold. Then, the densities $n$ and $p$ are bounded in $L^{\infty}(\Omega)$, uniformly as $\lambda \rightarrow 0$.

Proof. Consider

$$
\bar{C}=\operatorname{ess} \sup _{\Omega} C, \quad \underline{C}=\operatorname{essinf}_{\Omega} C .
$$

Then the maximum principle implies the estimates

$$
\ln \left(\frac{\underline{C}+\sqrt{\underline{C}^{2}+4 n_{i}^{2}}}{2 n_{i}}\right) \leqslant \varphi \leqslant \ln \left(\frac{\bar{C}+\sqrt{\bar{C}^{2}+4 n_{i}^{2}}}{2 n_{i}}\right),
$$

and, consequently,

$$
n \leqslant \frac{\bar{C}+\sqrt{\bar{C}^{2}+4 n_{i}^{2}}}{2}, \quad p \leqslant \frac{-\underline{C}+\sqrt{\underline{C}^{2}+4 n_{i}^{2}}}{2} .
$$

The uniform boundedness of $n_{i}$ remains to be proven. Setting

$$
\Omega_{1}=\{x \in \Omega: \varphi(x) \geqslant 0\}, \quad \Omega_{2}=\{x \in \Omega: \varphi(x) \leqslant 0\},
$$

we have $\left|\Omega_{1}\right|+\left|\Omega_{2}\right| \geqslant|\Omega|$, and

$$
\max \left\{\int_{\Omega} \mathrm{e}^{\varphi} \mathrm{d} x, \int_{\Omega} \mathrm{e}^{-\varphi} \mathrm{d} x\right\} \geqslant \max \left\{\left|\Omega_{1}\right|,\left|\Omega_{2}\right|\right\} \geqslant \frac{|\Omega|}{2},
$$


with the consequence

$$
n_{i} \leqslant \frac{2}{|\Omega|} \max \{N, P\}
$$

Note that the assumption (1.7) has not been used yet and that no uniform bound for the potential has been established so far. For this, we use a slightly strengthened version of condition (1.7):

$$
N>\int_{\Omega} C_{+} \mathrm{d} x, \quad P>\int_{\Omega} C_{-} \mathrm{d} x .
$$

We recall that the two inequalities are equivalent under assumption (1.1).

LEMMA 2.3 Let the assumptions of Theorem 2.1 and (2.4) hold. Then the potential $\varphi$ is bounded in $L^{\infty}(\Omega)$, uniformly as $\lambda \rightarrow 0$.

Proof. Considering (2.2) and (2.3), it is sufficient to prove that $n_{i}$ is uniformly bounded away from zero. We shall use

$$
\begin{aligned}
& N=\int_{\varphi \leqslant 0} n \mathrm{~d} x+\int_{\varphi>0} n \mathrm{~d} x \leqslant n_{i}\left|\Omega_{2}\right|+\int_{\varphi>0} n \mathrm{~d} x \leqslant n_{i}|\Omega|+\int_{\varphi>0} n \mathrm{~d} x, \\
& \int_{\varphi>0} p \mathrm{~d} x \leqslant n_{i}\left|\Omega_{1}\right| \leqslant n_{i}|\Omega|, \\
& \int_{\varphi>0} C \mathrm{~d} x \leqslant \int_{\varphi>0} C_{+} \mathrm{d} x \leqslant \int_{\Omega} C_{+} \mathrm{d} x, \quad \text { and } \\
& \int_{\varphi>0} \Delta \varphi \mathrm{d} x \leqslant 0 .
\end{aligned}
$$

Integration of the Poisson equation over $\{\varphi>0\}$ leads to the inequality

$$
0 \geqslant \int_{\varphi>0}(n-p-C) \mathrm{d} x \geqslant N-\int_{\Omega} C_{+} \mathrm{d} x-2|\Omega| n_{i},
$$

implying

$$
n_{i} \geqslant \frac{N-\int_{\Omega} C_{+} \mathrm{d} x}{2|\Omega|} .
$$

The Poisson equation (1.4) is singularly perturbed. So it is not a surprise that, for passing to the limit, we need a smoothness assumption on the data, i.e., the doping profile, as follows.

THEOREM 2.4 Let the assumptions of Theorem 2.1, (2.4), and $C \in H^{1}(\Omega)$ hold. Then, as $\lambda \rightarrow 0$ the solution $\varphi$ of (1.3)-(1.5) converges strongly in $L^{p}(\Omega)$ for every $p<\infty$ to the unique solution of the formal limiting problem.

Proof. Multiplication of the Poisson equation (1.4) by $n-p-C$, integration by parts, and using $\nabla n=n \nabla \varphi, \nabla p=-p \nabla \varphi$ gives

$$
\lambda^{2} \int_{\Omega}(n+p)|\nabla \varphi|^{2} \mathrm{~d} x+\int_{\Omega}(n-p-C)^{2} \mathrm{~d} x=\lambda^{2} \int_{\Omega} \nabla \varphi \cdot \nabla C \mathrm{~d} x .
$$


Note that, by Lemma 2.3, the densities are uniformly bounded away from zero: $n+p \geqslant c>0$. This implies uniform boundedness of $\varphi$ in $H^{1}(\Omega)$ and the strong convergence of $n-p-C$ to zero in $L^{2}(\Omega)$. By the $H^{1}(\Omega)$-bound, we have strong convergence (of a subsequence) of $\varphi$ to $\varphi_{0}$, and we can pass to the limit in the nonlinear terms, since $\varphi$ is also uniformly in $L^{\infty}(\Omega)$ as $\lambda \rightarrow 0$. It remains to prove uniqueness for the limiting problem

$$
0=n_{i 0} \mathrm{e}^{\varphi_{0}}-n_{i 0} \mathrm{e}^{-\varphi_{0}}-C, \quad n_{i 0}=\frac{N}{\int_{\Omega} \exp \left(\varphi_{0}\right) \mathrm{d} x} .
$$

Substitution of the solution $\varphi_{0}$ of the first equation into the second gives an equation for $n_{i 0}$ :

$$
\int_{\Omega}\left(C+\sqrt{C^{2}+4 n_{i 0}^{2}}\right) \mathrm{d} x=2 N
$$

having a unique solution, if (2.4) is satisfied.

\section{A limiting free boundary problem}

If the assumption (2.4) is violated, the potential is expected to take $O\left(\lambda^{-2}\right)$-values. Therefore we consider in this section the problem for the rescaled potential $\Phi=\lambda^{2} \varphi$ :

$$
\begin{aligned}
& \Delta \Phi=n-p-C, \quad \frac{\partial \Phi}{\partial v}=0, \text { on } \partial \Omega, \\
& n=\frac{N \exp \left(\Phi / \lambda^{2}\right)}{\int_{\Omega} \exp \left(\Phi / \lambda^{2}\right) \mathrm{d} x}, \quad p=\frac{P \exp \left(-\Phi / \lambda^{2}\right)}{\int_{\Omega} \exp \left(-\Phi / \lambda^{2}\right) \mathrm{d} x} .
\end{aligned}
$$

From the previous section we know that a solution exists, which is unique up to a shift in the potential $\Phi$, and that the densities $n$ and $p$ are bounded uniformly as $\lambda \rightarrow 0$. With the normalization $\min _{\Omega} \Phi=0$ of the potential, the Poisson equation (3.1) implies uniform boundedness of $\Phi$ in $W^{2, p}(\Omega)$ for every $p<\infty$ and, as a consequence, convergence (subsequencewise) of $\Phi$ to $\Phi_{0}$ in $C^{1}(\bar{\Omega})$. The densities $n$ and $p$ converge (after extracting a further subsequence) to $n_{0}$ and, respectively, $p_{0}$ in $L^{\infty}(\Omega)$ weak*. By the above, $\Phi$ is uniformly Lipschitz continuous. Introducing $\bar{\Phi}:=\max _{\Omega} \Phi=\Phi\left(x_{0}\right)$, we therefore have $\bar{\Phi}-\Phi(x) \leqslant L\left|x-x_{0}\right|$ and, thus,

$$
\int_{\Omega} \exp \left(\frac{\Phi-\bar{\Phi}}{\lambda^{2}}\right) \mathrm{d} x \geqslant \int_{\Omega} \exp \left(-\frac{L\left|x-x_{0}\right|}{\lambda^{2}}\right) \mathrm{d} x \geqslant c \lambda^{2 d}
$$

with a positive, $\lambda$-independent constant $c$. For the electron density, this implies

$$
n=\frac{N \exp \left(\frac{\Phi-\bar{\Phi}}{\lambda^{2}}\right)}{\int_{\Omega} \exp \left(\frac{\Phi-\bar{\Phi}}{\lambda^{2}}\right) \mathrm{d} x} \leqslant \frac{N}{c \lambda^{2 d}} \exp \left(\frac{\Phi-\bar{\Phi}}{\lambda^{2}}\right) .
$$

Assume $\bar{\Phi}_{0}=\max _{\Omega} \Phi_{0}>0$. Then the above estimate implies $n_{0}=0$ wherever $\Phi_{0}<\bar{\Phi}_{0}$ with uniform convergence of the electron density in compact subsets of $\left\{\Phi_{0}<\bar{\Phi}_{0}\right\}$. Analogously it is shown that $p$ converges to zero uniformly in compact subsets of $\left\{\Phi_{0}>0\right\}$. At this point it is clear from the theory of variational inequalities [8] that $\Phi_{0}$ solves a classical double obstacle problem 
with the obstacles $\Phi_{0}=0$ and $\Phi_{0}=\bar{\Phi}_{0}$, which can be written as the variational inequality that we find $\Phi_{0} \in \mathbb{K}=\left\{\Psi \in H^{1}(\Omega): 0 \leqslant \Psi \leqslant \bar{\Phi}_{0}\right\}$ such that

$$
\int_{\Omega}\left(\nabla \Phi_{0} \cdot \nabla\left(\Psi-\Phi_{0}\right)-C\left(\Psi-\Phi_{0}\right)\right) \mathrm{d} x \geqslant 0, \quad \forall \Psi \in \mathbb{K} .
$$

The domain $\Omega$ can be split in the form $\Omega=\Omega_{+} \cup \Omega_{-} \cup \mathcal{N}$, with the coincidence sets $\Omega_{+}=\{x \in$ $\left.\Omega: \Phi_{0}(x)=\bar{\Phi}_{0}\right\}, \Omega_{-}=\left\{x \in \Omega: \Phi_{0}(x)=0\right\}$, and the non-coincidence set $\mathcal{N}=\{x \in \Omega: 0<$ $\left.\Phi_{0}(x)<\bar{\Phi}_{0}\right\}$. From our previous results and from the limit of the Poisson equation (3.1) we have

$$
\begin{aligned}
& n_{0}=C, \quad p_{0}=0, \quad \text { in } \Omega_{+}, \\
& p_{0}=-C, \quad n_{0}=0, \quad \text { in } \Omega_{-}, \\
& n_{0}=p_{0}=0, \quad \Delta \Phi_{0}+C=0, \quad \text { in } \mathcal{N} .
\end{aligned}
$$

Fixing the value of $\bar{\Phi}_{0}$ would make the solution of the obstacle problem unique. By passing to the limit in the equation $\int_{\Omega} n \mathrm{~d} x=N$, we obtain

$$
\int_{\Omega_{+}} C \mathrm{~d} x=N
$$

It is clear from the above that in the coincidence set $\Omega_{+}$the doping profile takes non-negative values. The above equation, thus, implies

$$
N \leqslant \int_{\Omega} C_{+} \mathrm{d} x
$$

the complementary condition to (2.4). We recall that we assumed $\bar{\Phi}_{0}>0$, to arrive at (3.5). Therefore (2.4) implies $\bar{\Phi}_{0}=0$ and, consequently, $\Phi_{0} \equiv 0$ in $\Omega$, which is also a consequence of the analysis of the preceding section and of the rescaling $\Phi=\lambda^{2} \varphi$.

Note that (3.4) is equivalent to $\int_{\Omega_{-}} C \mathrm{~d} x=-P$. This follows from the global neutrality condition and from the fact that $\Phi_{0}$ solves a Neumann problem in $\mathcal{N}$ implying $\int_{\mathcal{N}} C \mathrm{~d} x=0$.

It remains to show that $\bar{\Phi}_{0}$ is uniquely determined by the side condition (3.4). We first show that the dependence of the coincidence sets on $\bar{\Phi}_{0}$ is monotone. Let $\alpha, \beta$ with $0<\alpha<\beta$ be two different values for $\bar{\Phi}_{0}$ and denote by $\Phi^{\alpha}, \Phi^{\beta}$ the corresponding solutions of (3.3). We shall also need the corresponding coincidence and non-coincidence sets $\Omega_{-}^{\alpha}, \Omega_{+}^{\alpha}, \mathcal{N}^{\alpha}, \Omega_{-}^{\beta}, \Omega_{+}^{\beta}, \mathcal{N}^{\beta}$. Then $\Phi^{\alpha}$ is the solution of a single obstacle problem $\left(\Phi^{\alpha} \geqslant 0\right)$, when $C$ is replaced by

$$
f= \begin{cases}C & \text { in } \Omega_{-}^{\alpha} \cap \mathcal{N}^{\alpha}, \\ 0 & \text { in } \Omega_{+}^{\alpha} .\end{cases}
$$

Now we define $\tilde{\Omega}=\left\{x \in \Omega: \Phi^{\beta}(x)<\alpha\right\}$. Then, in $\tilde{\Omega}, \Phi^{\beta} \geqslant 0$ is a supersolution of $\Delta \Phi+f=0$. Also, $\frac{\partial \Phi^{\beta}}{\partial v}=0$ or $\Phi^{\beta}=\alpha \geqslant \Phi^{\alpha}$ on $\partial \tilde{\Omega}$. By a slight generalization of Theorem II.6.4 in [8] (to mixed boundary conditions), $\Phi^{\alpha} \leqslant \Phi^{\beta}$ holds and, thus, $\Omega_{-}^{\beta} \subset \Omega_{-}^{\alpha}$. Similarly, $\Omega_{+}^{\beta} \subset \Omega_{+}^{\alpha}$ is shown.

Assume that

$$
\int_{\Omega_{+}^{\alpha}} C \mathrm{~d} x=\int_{\Omega_{+}^{\beta}} C \mathrm{~d} x \quad\left(\Longleftrightarrow \int_{\Omega_{-}^{\alpha}} C \mathrm{~d} x=\int_{\Omega_{-}^{\beta}} C \mathrm{~d} x\right) .
$$


By $C \geqslant 0$ in $\Omega_{+}^{\alpha}\left(C \leqslant 0\right.$ in $\left.\Omega_{-}^{\alpha}\right)$, this implies

$$
C=0 \text { a.e. in }\left(\Omega_{+}^{\alpha} \backslash \Omega_{+}^{\beta}\right) \cup\left(\Omega_{-}^{\alpha} \backslash \Omega_{-}^{\beta}\right) .
$$

From the consequence $\Delta\left(\Phi^{\alpha}-\Phi^{\beta}\right)=0$ in $\Omega$ and from $\Phi^{\alpha}=\Phi^{\beta}=0$ in $\Omega_{-}^{\beta}$ we deduce the contradiction $\Phi^{\alpha}=\Phi^{\beta}$ in $\Omega$ to $\Phi^{\alpha}=\alpha<\beta=\Phi^{\beta}$ in $\Omega_{+}^{\beta}$. Therefore

$$
\int_{\Omega_{+}^{\alpha}} C \mathrm{~d} x>\int_{\Omega_{+}^{\beta}} C \mathrm{~d} x
$$

and the map $\bar{\Phi}_{0} \mapsto \int_{\Omega_{+}} C \mathrm{~d} x$ is strictly monotone.

THEOREM 3.1 Let the assumptions of Theorem 2.1 and (3.5) hold. Then, as $\lambda \rightarrow 0$ the solution $\Phi$ of (3.1), (3.2) converges in $C^{1}(\bar{\Omega})$ to the unique solution of the variational inequality (3.3) where the upper obstacle $\bar{\Phi}_{0}$ is uniquely determined by (3.4).

Finally, a variational formulation of the limiting problem will be given. We start with a rescaled version of the energy functional introduced at the beginning of the preceding section:

$$
\begin{aligned}
J_{\lambda}(\Psi)= & \lambda^{2} J\left(\frac{\Psi}{\lambda^{2}}\right)=\int_{\Omega}\left(\frac{1}{2}|\nabla \Psi|^{2}-C \Psi\right) \mathrm{d} x \\
& +\lambda^{2} N \ln \left(\int_{\Omega} \mathrm{e}^{\Psi / \lambda^{2}} \mathrm{~d} x\right)+\lambda^{2} P \ln \left(\int_{\Omega} \mathrm{e}^{-\Psi / \lambda^{2}} \mathrm{~d} x\right) .
\end{aligned}
$$

The second term on the right-hand side can be rewritten in the form

$$
N \sup _{\Omega} \Psi+\lambda^{2} N \ln \left(\int_{\Omega} \exp \left(\frac{\Psi-\sup _{\Omega} \Psi}{\lambda^{2}}\right) \mathrm{d} x\right) .
$$

Formally passing to the limit in $J_{\lambda}$ for $\Psi \in \mathbb{K}_{0}=\left\{\Psi \in H^{1}(\Omega): \Psi \geqslant 0\right\}$ gives

$$
J_{0}(\Psi)=\int_{\Omega}\left(\frac{1}{2}|\nabla \Psi|^{2}-C \Psi\right) \mathrm{d} x+N \sup _{\Omega} \Psi .
$$

It is easily shown that $J_{0}$ is bounded from below, weakly lower semi-continuous, and strictly convex on $\mathbb{K}_{0}$. We shall show that the unique minimizer $\Psi_{0} \in \mathbb{K}_{0}$ of $J_{0}$ is equal to $\Phi_{0}$. First, by fixing $\sup _{\Omega} \Psi_{0}$, it is clear that $\Psi_{0}$ solves a double obstacle problem. Second, we compute

$$
0=\left.\frac{\mathrm{d}}{\mathrm{d} \mu} J_{0}\left(\mu \Psi_{0}\right)\right|_{\mu=1}=\int_{\Omega}\left(\left|\nabla \Psi_{0}\right|^{2}-C \Psi_{0}\right) \mathrm{d} x+N \sup _{\Omega} \Psi_{0} .
$$

Only the coincidence set where $\Psi_{0}=\sup _{\Omega} \Psi_{0}$ contributes to the integral, showing that (3.6) is equivalent to (3.4).

\section{Acknowledgements}

This work has been supported by the TMR network No. ERB FMRX CT97 0157 and by the Erwin Schrödinger Institute, Wien. Luis Caffarelli also acknowledges support by the NSF under grant no. 9714758. 


\section{REFERENCES}

1. BRezzi, F., CAPElo, A., \& GASTALDi, L. A singular perturbation analysis of reverse biased semiconductor diodes. SIAM J. Math. Anal. 20, (1989) 372-387.

2. CAfFARElli, L. \& Friedman, A. A singular perturbation problem in semiconductors. Bolletino U.M.I. $1-B$ 7, (1987) 409-421.

3. Desvillettes, L. \& Dolbeault, J. On long time asymptotics of the Vlasov-PoissonBoltzmann equation. Comm. Partial Differential Equations 16(2, 3), (1991) 451-489.

4. Dolbeault, J. Stationary states in plasma physics: Maxwellian solutions of the Vlasov-Poisson system. Math. Models and Meth. in the Appl. Sci. 1, (1991) 183-208.

5. Gogny, D. \& Lions, P.-L. Sur les états d'équilibre pour les densités électroniques dans les plasmas. RAIRO Modél. Math. Anal. Numér. 23(1), (1989) 137-153.

6. HunT, C. \& NASSIF, N. R. On a variational inequality and its approximation, in the theory of semiconductors. SIAM J. Numer. Anal. 12, (1975) 938-950.

7. JÜNGEL, A. The free boundary problem of a semiconductor in thermal equilibrium. Math. Meth. in the Appl. Sci. 18, (1995) 387-412.

8. Kinderlehrer, D. \& Stampacchia, G. An Introduction to Variational Inequalities and Their Applications. Academic Press, New York (1980).

9. MARKOWICH, P. A. A singular perturbation analysis of the fundamental semiconductor device equations. SIAM J. Appl. Math. 44, (1984) 896-928.

10. Markowich, P. A., Ringhofer, C., \& Schmeiser, C. Semiconductor Equations. Springer-Verlag, Wien (1990).

11. Markowich, P. A. \& Unterreiter, A. Vacuum solutions of a stationary drift-diffusion model. Ann. Scuola Norm. Sup. Pisa 20, (1993) 371-386.

12. Montarnal, Ph. \& Perthame, B. Analyse asymptotique des équations de dérive diffusion et équations de Hamilton-Jacobi. Rapport de Recherche INRIA, No. 2885. (1996)

13. Montarnal, Ph. \& Perthame, B. Asymptotic analysis of the drift-diffusion equations and Hamilton-Jacobi equations. Math. Mod. and Meth. in Appl. Sci. 7, (1997) 61-80.

14. O’Malley, R. E. \& Schmeiser, C. The asymptotic solution of a semiconductor device problem involving reverse bias. SIAM J. Appl. Math. 50, (1990) 504-520.

15. SCHMEISER, C. On strongly reverse biased semiconductor diodes. SIAM J. Appl. Math. 49, (1989) 17341748.

16. SCHMEISER, C. A singular perturbation analysis of reverse biased pn-junctions. SIAM J. Math. Anal. 21 , (1990) 313-326.

17. Shockley, W. The theory of $\mathrm{p}-\mathrm{n}$ junctions in semiconductors and $\mathrm{p}-\mathrm{n}$ junction transistors. Bell Syst. Tech. J. 28, (1949) 435.

18. Unterreiter, A. The thermal equilibrium state of semiconductor devices. Appl. Math. Lett. 7, (1994) 39-43.

19. UNTERREITER, A. The thermal equilibrium solution of a generic bipolar quantum hydrodynamic model. Comm. Math. Phys. 188, (1997) 69-88. 